\title{
Quantitative Determination of Irinotecan and the Metabolite SN-38 by Nanoflow Liquid Chromatography-Tandem Mass Spectrometry in Different Regions of Multicellular Tumor Spheroids
}

\author{
Xin Liu, Amanda B. Hummon
}

Department of Chemistry and Biochemistry, Harper Cancer Research Institute, University of Notre Dame, 251 Nieuwland Science Hall, Notre Dame, IN 46556, USA

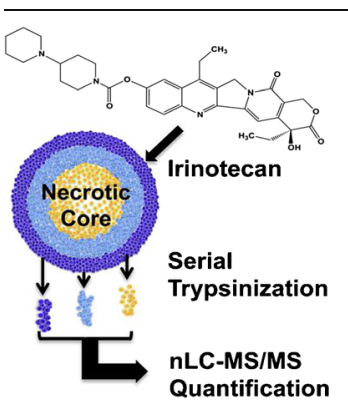

Abstract. A new and simple method was developed to evaluate the distribution of therapeutics in three-dimensional multicellular tumor spheroids (MCTS) by combining serial trypsinization and nanoflow liquid chromatography-tandem mass spectrometry (nLC-MS/MS). This methodology was validated with quantitative measurements of irinotecan and its bioactive metabolite, SN-38, in distinct spatial regions of HCT 116 MCTS. Irinotecan showed a time-dependent permeability into MCTS with most of the drug accumulating in the core after $24 \mathrm{~h}$ of treatment. The amount of SN-38 detected was 30 times lower than that of the parent drug, and was more abundant in the outer rim and intermediate regions of MCTS where proliferating cells were present. This method can be used to investigate novel and established drugs. It enables investigation of drug penetration properties and identification of metabolites with spatial specificity in MCTS. The new approach has great value in facilitating the drug evaluation process.

Key words: nLC-MS/MS, 3D cell cultures, Serial trypsinization, Irinotecan, SN-38, Multicellular tumor spheroids

Received: 2 September 2014/Revised: 20 November 2014/Accepted: 17 December 2014/Published Online: 21 January 2015

\section{Introduction}

$\mathrm{T}$ he invention, development, and evaluation of a new drug is a slow, expensive, and failure-prone process. Over the past $15 \mathrm{y}$, although there has been an increase in the number of potential therapeutic agents under development, the number of molecules that successfully progress through clinical trials is low $[1,2]$. Lack of a quantitative understanding of drug uptake and diffusion within tissues is one of the many causes of drug failure [3-6]. The ability for a drug to cross biological barriers like the plasma membrane, cell adhesion molecules, and cellular efflux pumps is a key determinant of its absorption, distribution, metabolism, and excretion (ADME) in the human body $[5,7]$. It is, therefore, critical to improve preclinical in vitro cell-based testing approaches to sieve out poorly penetrating compounds and prioritize promising candidates.

Electronic supplementary material The online version of this article (doi:10.1007/s13361-014-1071-0) contains supplementary material, which is available to authorized users.

Correspondence to: Amanda Hummon; e-mail: ahummon@nd.edu
To produce reliable biomedically relevant information from an in vitro assay for drug assessment, it is necessary that the cells used in the analysis mimic the phenotype of cells within the in vivo target tissue [8]. Twodimensional (2D) cell culture (i.e., monolayer or suspension culture) is conventionally used with in vitro drug candidate testing, but it does not accurately resemble the complexity found in a three-dimensional (3D) multicellular environment [8-10]. The uptake and diffusion distance for a drug into monolayer cells is relatively short compared with in vivo tissues, and the biological barriers are not adequately mimicked [6, 9, 11, 12].

To bridge the gap between in vitro and in vivo therapeutic evaluations, Sutherland and coworkers first described in vitro multicellular tumor spheroids (MCTS) in 1971 [13]. The MCTS model is a 3D spheroidal cluster of cells that replicates heterogeneous, avascular tumor masses [10-13]. A MCTS consists of chemical diffusion gradients that result in distinct cellular microenvironments $[3,6]$. For example, highly proliferative cells are located at the outer rim of the MCTS and quiescent or necrotic cells are located in the core region [8]. 
The current analytical methods to study the drug and drug metabolite distributions in MCTS tissue mimics are cumbersome and limited $[5,6,9]$. Microscopy techniques have been developed for quantitative measurements of drug concentrations in MCTS for therapeutics having sufficient color. Fluorescent compounds like doxorubicin [14-16] and mitoxantrone [17] can also be directly analyzed with fluorescence microscopy. The distribution of other radiolabeled drugs has also been characterized by autoradiography [18-20]. However, for molecules without fluorescence or radiolabels, detecting the drug and associated metabolites is challenging $[5,6]$.

We previously established a matrix-assisted laser desorption/ionization (MALDI) imaging mass spectrometry (IMS) method to map the localization of drugs and their metabolites in MCTS [21]. This approach successfully enabled chemical analysis in a spatially defined manner, but quantification of specific analytes is difficult. Also, the sample preparation protocol for the imaging approach is relatively timeconsuming, resulting in lower throughput for batch analysis. Serial trypsinization is an alternative and simpler experimental method that also allows cells from MCTS to be analyzed with spatial specificity [22]. In this procedure, a short trypsin treatment is used to sequentially remove cells from the outside of a spheroid in concentric radial layers, analogous to "peeling an onion." In a previous study employing serial trypsinization, the isolated fractions of cells from surface layers, the intermediate region, and the necrotic core of MCTS were analyzed with quantitative iTRAQ chemistry to examine differences in the protein expression profiles in distinct spatial areas of MCTS [23]. We also previously employed serial trypsinization in combination with single cell capillary electrophoresis to study the metabolism of glycosphingolipids in different regions of MCTS [24].

In this report, we applied serial trypsinization to map the distribution of exogenously applied compounds. We treated MCTS with an exogenous therapeutic and then analyzed the concentric cell layers by nLC-MS/MS to evaluate the molecular distribution. As an initial proof-of principle study, we first evaluated the ability of irinotecan \{CPT-11, 7-ethyl-10- [4-(1piperidino)-1-piperidino]-carbonyloxy camptothecin, Figure 1a\} to penetrate the MCTS, as we have previously mapped its distribution via imaging mass spectrometry. Irinotecan has been widely used for the treatment of colorectal cancer and other solid tumors for both first line and second line therapy [25]. In humans, irinotecan is a prodrug that is hydrolyzed by carboxylesterases to form its highly active metabolite, $\mathrm{SN}-38$ (7-Ethyl-10-hydroxycamptothecin, Figure 1b), which exerts its cytotoxic effects by inhibiting the activity of DNA topoisomerase I (Topo I) [26, 27]. We also evaluated the distribution of SN-38 in the MCTS via nLC-MS/MS. Camptothecin \{CPT, (4S)-4-Ethyl-4-hydroxy-1H-pyrano $\left[3^{\prime}, 4^{\prime}: 6,7\right]$ indolizino [1,2-b]quinoline-3,14(4H,12H)-dionequinoline-3,14$(4 \mathrm{H}, 12 \mathrm{H})$-dione, Figure $1 \mathrm{c}\}$, an analogue of irinotecan, was used as an internal standard. Several methods have been developed for simultaneous measurement of irinotecan and SN-38 in biological fluids [28-35], but a quantitative investigation of their in vitro spatial distribution in tumor mimics provides valuable insight into the ability of this drug to penetrate cell masses.

\section{Methods}

\section{Cell Culture and Growth of the MCTS}

The human colon carcinoma cell line HCT 116 was obtained from American Type Culture Collection (ATCC, Manassas, VA, USA) and maintained in McCoy's 5A cell culture medium (Life Technologies, Grand Island, NY, USA) supplemented with $10 \%$ fetal bovine serum (Thermo Scientific, Gaithersburg, MD, USA) and $2.5 \mathrm{mM}$ L-glutamine (Invitrogen, San Diego, CA, USA). The cell line was used within 3 mo of resuscitation of frozen aliquots thawed from liquid nitrogen. The provider assured authentication of the cell line by cytogenetic analysis. MCTS cell culture was carried out using agarose-coated 96well plates by seeding 7000 cells into the middle of each well as previously described $[21,36]$. Half the volume of the culture medium was changed every $48 \mathrm{~h}$ after $4 \mathrm{~d}$ in culture. The MCTS reached a diameter of $\sim 1 \mathrm{~mm}$ after $12 \mathrm{~d}$ in culture.

\section{Drug Treatment, MCTS Fractionation, and Preparation for $n L C-M S / M S$ Analysis}

Irinotecan hydrochloride and camptothecin were purchased from Sigma (St. Louis, MO, USA). Stock solutions of irinotecan and camptothecin were prepared in nanoPure water and dimethyl sulfoxide (DMSO), respectively, at a concentration of $0.5 \mathrm{mg} / \mathrm{mL}$. The irinotecan solution was further diluted in McCoy's 5A medium to achieve a final concentration of the previously determined $\mathrm{IC}_{50}, 20.6 \mu \mathrm{M}$ [21]. Two hundred microliters of this solution was then added to the $1 \mathrm{~mm}$ diameter MCTS by replacing the old medium. These MCTS were treated for various lengths of time $(1,2,6,12,24,48$, and $72 \mathrm{~h})$ and the same volume of medium without the drug was added to the control MCTS. After specific incubation times, 20 MCTS were harvested and washed three times with ice-cold $1 \mathrm{X}$ phosphate buffered saline (PBS).

Cells from these MCTS were separated layer by layer using serial trypsinization [22-24]. A solution of $0.05 \%$ trypsin/ EDTA at room temperature was added, and the MCTS were gently rotated for $3 \mathrm{~min}$ on a rotary shaker at approximately $60 \mathrm{rpm}$. Chilled complete medium was then added to inhibit the trypsin. The cell suspension was carefully collected from the MCTS, and medium without FBS was added to wash serum out of the dish to allow further trypsin proteolysis. By repeating the 3 min trypsinization, complete medium washing, and serum-free medium washing steps repeatedly, cells from progressively deeper regions in the MCTS were harvested. The first three fractions were grouped as a single sample representing the outer rim of MCTS. Cells from the next four trypsin treatments were collected representing the intermediate region. The eighth fraction contained the remaining spheroid necrotic core, which was dissociated completely by pipetting. Cell 
(a)

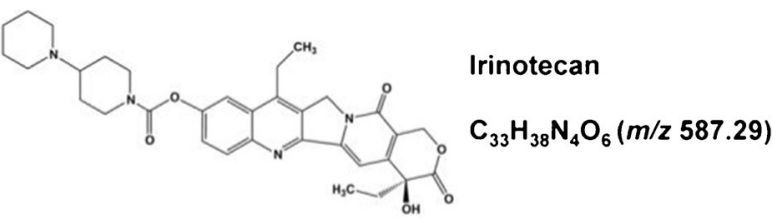

(b)

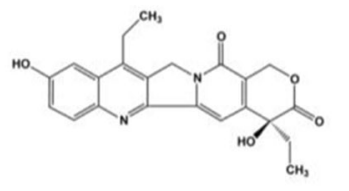

SN-38

$\mathrm{C}_{22} \mathrm{H}_{20} \mathrm{~N}_{2} \mathrm{O}_{5}(\mathrm{~m} / 2$ 393.14)

(c)

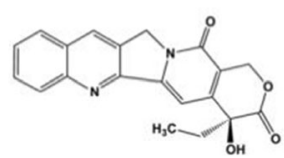

Camptothecin

$\mathrm{C}_{20} \mathrm{H}_{16} \mathrm{~N}_{2} \mathrm{O}_{4}(\mathrm{~m} / \mathrm{z} 349.12)$

Figure 1. Chemical structures of (a) irinotecan, (b) SN-38, and (c) the internal standard, camptothecin (CPT)

suspensions from each of the populations were centrifuged at $2000 \mathrm{~g}$ for $10 \mathrm{~min}$ and cell pellets were washed twice with PBS. The weights of the trypsinized cells from different regions of MCTS were measured on a balance. Small molecules were then extracted by adding $500 \mu \mathrm{L}$ extraction solution $(0.01 \mathrm{M}$ HCl:methanol, 2:3, v/v) containing $200 \mathrm{nM}$ camptothecin. After mixing by vortex, the cellular extracts were sonicated and centrifuged at $15,000 \mathrm{~g}$ for $15 \mathrm{~min}$ at $4^{\circ} \mathrm{C}$. The supernatants were transferred into clean microtubes and evaporated to dryness under vacuum using a Speedvac. The dried extracts were redissolved by vortexing in $100 \mu \mathrm{L}$ of $20: 80(\mathrm{v} / \mathrm{v})$ methanol:water solution containing $0.1 \%$ acetic acid and $2 \mu \mathrm{L}$ was then injected directly into the nLC-MS/MS system for analysis. To analyze metabolites efflux, $20 \mu \mathrm{L}$ cell culture medium after incubation of MCTS with irinotecan was collected at different time points and small molecules were then extracted using the same sample pretreatment procedure described above. All treatment conditions were performed in biological triplicate. A schematic illustration for this approach is shown in Figure 2.

\section{Calibration Curves and Method Validation}

SN-38 was purchased from Selleck Chemicals (Selleck, Houston, TX, USA). A standard stock solution $(0.5 \mathrm{mg} / \mathrm{mL})$ of SN38 was dissolved in DMSO. Working solutions for both irinotecan and SN-38 were prepared by serial dilutions with an icecold 0.01 M HCL:methanol mixture (2:3, v/v). All stock solutions were stored at $-80^{\circ} \mathrm{C}$ under dark conditions to remain stable for more than a month [31]. For drug analysis in MCTS, calibration samples were prepared by spiking irinotecan or $\mathrm{SN}$ 38 standards and camptothecin into drug-free control MCTS extracts $(500 \mu \mathrm{L})$. The mixture was subsequently evaporated to dryness and resuspended. The following concentrations were prepared in duplicate: irinotecan: $0.0025,0.025,0.25,2.5$, and $5 \mu \mathrm{M}$; SN-38: $0.0025,0.025,0.05,0.25$, and $0.5 \mu \mathrm{M}$, with a final camptothecin concentration of $1 \mu \mathrm{M}$. Medium calibration standards were prepared in drug-free medium extractions at

final concentrations of $0.005,0.025,0.25,1,2.5$, and $5 \mu \mathrm{M}$ for irinotecan; $0.005,0.025,0.25,0.5,1$, and $2.5 \mu \mathrm{M}$ for SN-38. The calibration curves were constructed by plotting the peak area of the analytes in the chromatogram over the camptothecin versus the concentrations spiked into the samples. The linearity was determined by calculation of a regression line using the method of least squares analysis.

Separate quality control (QC) samples were prepared independent of those used for preparing calibration curves at concentrations of: $0.0025,0.25$, and $5 \mu \mathrm{M}$ for irinotecan in spheroids lysates, and $0.005,0.25$, and $5 \mu \mathrm{M}$ in culture medium; $0.0025,0.25$, and $0.5 \mu \mathrm{M}$ for $\mathrm{SN}-38$ in spheroids lysates, and $0.005,0.25$, and $0.5 \mu \mathrm{M}$ in culture medium. They were prepared using the same procedure as the drug treated samples. Accuracy was determined by analysis of the signals in the chromatogram from both irinotecan and $\mathrm{SN}-38$ in five replicates on a single day and on three consecutive days, respectively, followed by a comparison with the calibration curves prepared on the same day and on different days.

The extraction efficiency from the MCTS cells or medium was determined by comparing the mean peak areas of extracted QC samples $(n=3)$ for irinotecan or for SN-38 to mean peak areas of these drugs in non-extracted samples by spiking in the same amounts of irinotecan or SN-38 at the step immediately prior to $\mathrm{nLC}-\mathrm{MS} / \mathrm{MS}$.

\section{nLC-MS/MS Conditions}

A nanoAcquity ultra performance LC system $(100 \mu \mathrm{m} \times 100 \mathrm{~mm}$ C18 BEH column) (Waters, Milford, MA, USA) was used for separation of the small molecules. Samples were eluted with a binary solvent system with $0.1 \%$ formic acid (A) and $0.1 \%$ formic acid in acetonitrile (B) using the following linear gradient separation: buffer B was increased from $3 \%$ to $60 \%$ in $15 \mathrm{~min}$, washed with $85 \%$ B for $5 \mathrm{~min}$, and equilibrated for $10 \mathrm{~min}$ with $3 \%$ buffer $\mathrm{B}$ before the next injection. The eluted small molecules were analyzed using a Q-Exactive instrument (Thermo Fisher Scientific, Waltham, MA, USA). Ionization source parameters were set as follows: spray voltage, $1.8 \mathrm{kV}$; capillary temperature, $280^{\circ} \mathrm{C}$; and s-lens level, 50.0. Full MS scans were operated over the range $\mathrm{m} / \mathrm{z} 330-800$ with a mass resolution of 70,000 with automatic gain control (AGC) target of 1.00E +06 . The 12 most intense peaks were fragmented in the HCD collision cell with normalized collision energy of $32 \%$, and tandem mass spectra were acquired with a mass resolution of 17,500 (AGC 1.00E + 05). The maximum allowed ion transfer time (IT) was $200 \mathrm{~ms}$ for full MS scans and $80 \mathrm{~ms}$ for tandem mass spectra.

The targeted multiplex SIM scans for quantification were acquired with AGC target value of $1.00 \mathrm{E}+05$, resolution of 70,000 , and maximum IT of $100 \mathrm{~ms}$ and the targets included irinotecan $(\mathrm{m} / z$ 587.28751), SN-38 $(\mathrm{m} / \mathrm{z} 393.14560)$, and camptothecin $(\mathrm{m} / \mathrm{z}$ 349.11938). Each target was monitored with a 2-min window (retention time $\pm 1 \mathrm{~min}$ ), 1-u isolation window (target $m / z \pm 0.5 \mathrm{u}$ ). Samples were run with duplicate injections. The data were analyzed and quantified by Xcalibur software (Thermo Fisher Scientific, Waltham, MA, USA). 


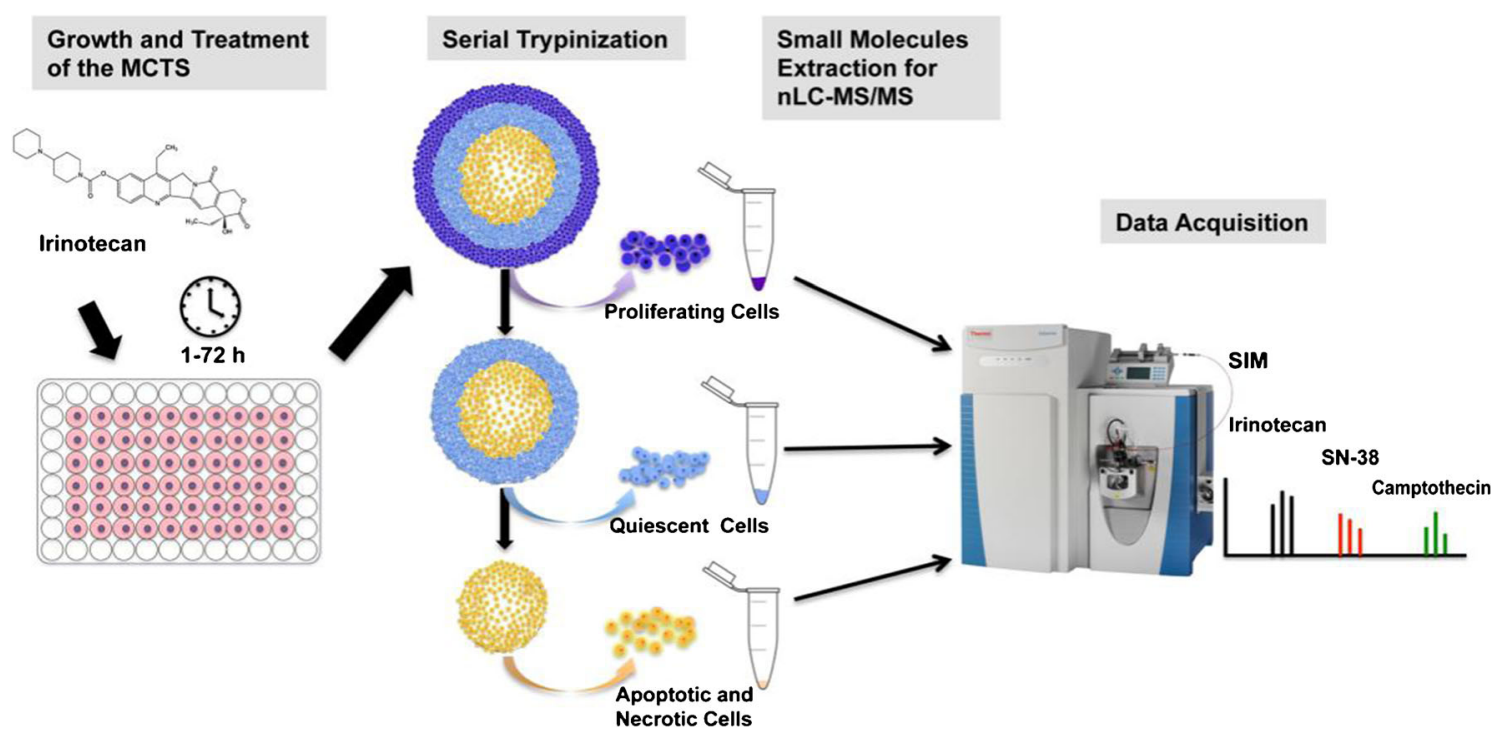

Figure 2. Schematic illustration of the experimental design

\section{Results and Discussion}

Multicellular tumor spheroids (MCTS) have gained popularity as an effective in vitro platform for testing drug delivery and metabolism in a cellular microenvironment that more closely mimics conditions in humans $[6,12]$. Some of these conditions include heterogeneous cell types, cell-cell, and cellular-matrix interactions, tight junctions, and chemical gradients. These physical characteristics limit drug penetration similar to solid tumors, tissues, and organs in vivo [6, 37]. However, the lack of simple and standardized assay techniques for the evaluation of drug delivery and efficacy in the spheroid model system has hindered its adoption into the mainstream drug screening process. In this report, we present a flexible method combining serial trypsinization and nLC-MS/MS to achieve measurements of drug permeability and metabolism in MCTS.

\section{MCTS Fractionation Using Serial Trypsinization}

Serial trypsinization was developed as a reproducible method that would uniformly isolate subpopulations of cells from increasing radial depth within the MCTS [22]. By dissociating MCTS into concentric layers and then into single cells, the relationship between drug uptake and metabolism in a specific cellular microenvironment can be studied. Optical images of a single spheroid during seven sequential trypsin treatments are shown in Supplemental Figure 1a. Cells were dissociated layer by layer without fracture of the remaining portion of the MCTS. The reproducibility of the approach was confirmed by measuring the weight of cells separated from outer surface layers, intermediate region, and the core area from 20 MCTS in 21 replicates (Supplemental Figure 1b). This procedure allows the investigation of differences in drug distribution between specific radial cellular locations in distinct spatial regions of MCTS.

\section{Method Development and Validation Specificity}

UPLC conditions were optimized with irinotecan, SN-38, and camptothecin standards. Different gradients were tested to obtain better separation and peak shape. The retention times for irinotecan, SN-38, and camptothecin were approximately $17.46,18.08$, and $18.27 \mathrm{~min}$, respectively, and full scan positive ion spectra for these compounds gave protonated species of $\mathrm{m} / \mathrm{z} 587.29,393.14$, and 349.12 , which match the calculated $[\mathrm{M}+\mathrm{H}]^{+}$molecular weights. By analyzing drug-free control spheroid lysates, or blank medium extracts, direct interference from endogenous peaks at or near the retention times of the analytes is minimal. Figure 3 shows typical chromatograms obtained from control MCTS, blank medium, irinotecan treated spheroids lysates, and drug containing medium, indicating sufficient separation amongst irinotecan, SN38 and camptothecin.

\section{Ion Suppression}

The matrix effect was investigated by comparing the peak areas of samples prepared in MCTS extracts or medium with the peak areas of neat solutions at the same concentrations. A small decrease in signal was observed for all analytes, but because the reduction in signal for the camptothecin and the other analytes coincided, the observed ion suppression was corrected when concentrations were calculated. The signal decreased by less than $15 \%$ for standards prepared in MCTS extracts, and by less than $20 \%$ when samples were prepared in medium. 
(a)

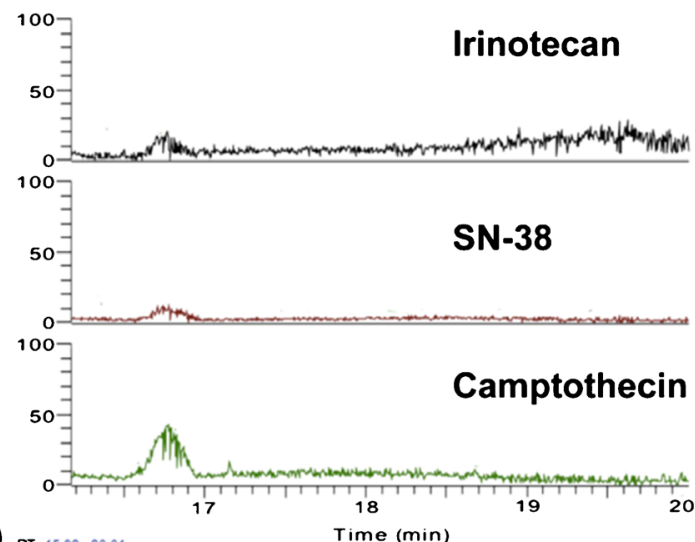

(c)

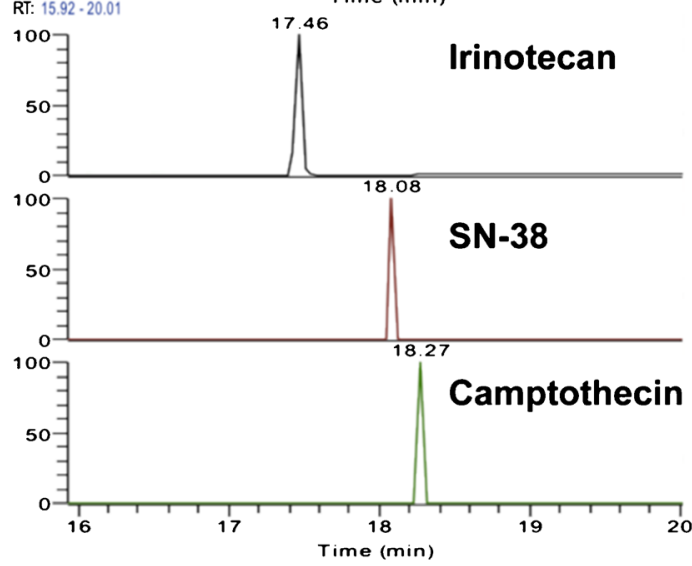

(b)

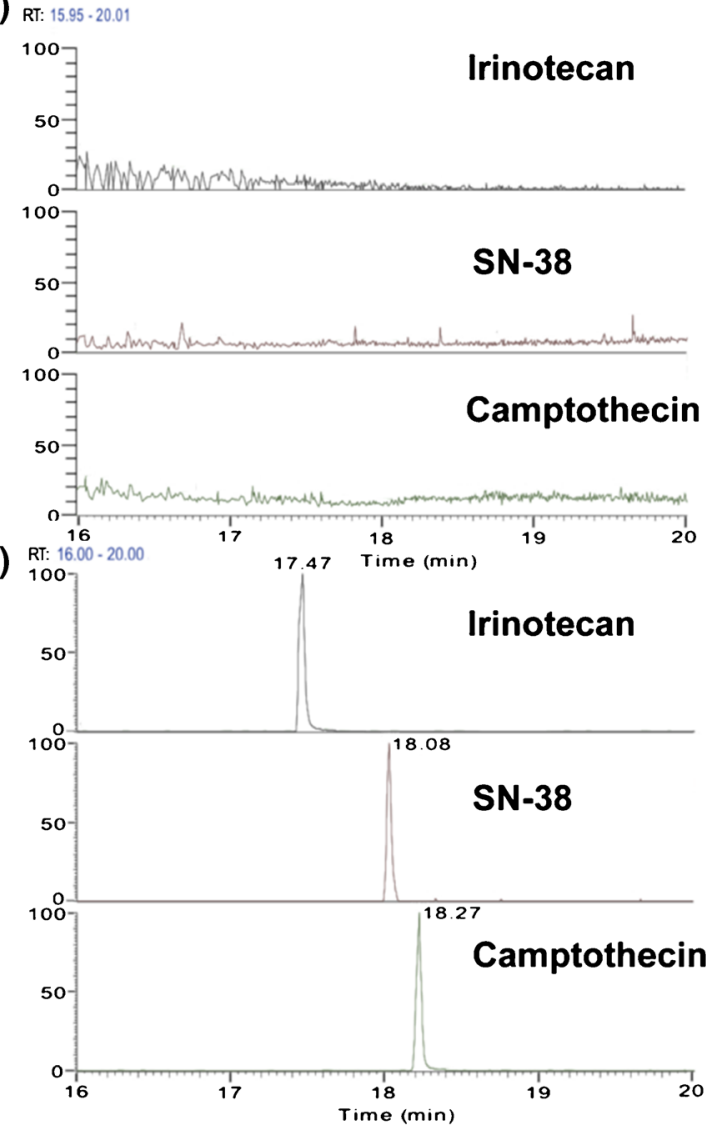

Figure 3. Representative chromatograms obtained from (a) untreated MCTS extracts, (b) blank culture medium extracts, (c) extracted sample from core region of irinotecan $(20.6 \mu \mathrm{M}, 72 \mathrm{~h})$ treated MCTS, and (d) extracted sample from culture medium collected $72 \mathrm{~h}$ after incubation of MCTS with irinotecan $(20.6 \mu \mathrm{M})$

\section{Linearity}

Calibration curves were constructed for both irinotecan and SN-38 in either MCTS extracts or medium. The calculated peak area ratios of calibration standards to camptothecin were proportional to the concentration of the analytes over selected concentration ranges: 0.0025-5 $\mu \mathrm{M}$ in MCTS lysates, 0.005$5 \mu \mathrm{M}$ in culture medium for irinotecan; $0.0025-0.5 \mu \mathrm{M}$ in MCTS lysates, and $0.005-2.5 \mu \mathrm{M}$ in culture medium for SN38 (Supplemental Figure 2). The linearity of the assay was determined by generation of a regression line using least square analysis. As evident from the coefficients of $\geq 0.99$ for all runs, calibration curves met acceptance criteria for both irinotecan and SN-38 at tested concentrations.

\section{Accuracy and Precision}

QC samples for each analyte were prepared in five replicates at three different concentrations (low, medium, and high) by independently weighing separate amounts of the standards used to construct calibration curves. The relative standard deviation (R.S.D.) ranges for irinotecan and $\mathrm{SN}-38$ in MCTS extracts were from $2.7 \%$ to $14.1 \%$ and from $7.9 \%$ to $12.5 \%$, respectively. The accuracy ranged from $101.1 \%$ to $108 \%$ and from $94 \%$ to $110.6 \%$ for irinotecan and SN-38. For samples in culture medium, R.S.D. for irinotecan and SN-38 ranged from $5.4 \%$ to $13 \%$, and from $6.2 \%$ to $14.4 \%$, respectively, and the accuracy ranges were from $86 \%$ to $97.8 \%$ and $90 \%$ to $105.4 \%$. Both precision (R.S.D. $\leq 15 \%)$ and accuracy $(85 \%-115 \%)$ were acceptable. The limit of quantification (LOQ) was defined as the lowest drug concentration that could be determined with acceptable precision (R.S.D. $\leq 20 \%)$ and accuracy $(80 \%-$ $120 \%$ ). The LOQ values were $2.5 \mathrm{nM}$ for irinotecan and SN38 in MCTS lysates; and $5 \mathrm{nM}$ in culture medium. The accuracy and precision data are shown in Table 1. Supplemental Figure 3 shows the recovery of the camptothecin at the concentration used in the method $(1 \mu \mathrm{M})$.

\section{Extraction Efficiency}

A protein precipitation method was used as the extraction procedure. The selection of appropriate solvents is a critical factor in this extraction approach. The extraction solvent needs to be miscible with water, stable during the extraction, and have high solubility for the target analytes. Different combinations of organic solvents and their mixtures with various polarities (acetonitrile, methanol, diethyl ether) were tested for extraction 
Table 1. Accuracy and Precision for the Analysis of Irinotecan and SN-38 ( $n=3$ days, five replicates per day)

\begin{tabular}{|c|c|c|c|c|c|c|c|}
\hline \multirow{2}{*}{$\begin{array}{l}\text { Nominal } \\
\text { concentration (nM) }\end{array}$} & \multicolumn{3}{|l|}{ MCTS extracts } & \multirow{2}{*}{$\begin{array}{l}\text { Nominal } \\
\text { concentration (nM) }\end{array}$} & \multicolumn{3}{|l|}{ Culture medium } \\
\hline & $\begin{array}{l}\text { Concentration } \\
(\text { mean } \pm \text { S.D. })(\mathrm{nM})\end{array}$ & Accuracy (\%) & $\begin{array}{l}\text { Precision } \\
\text { (R.S.D., \%) }\end{array}$ & & $\begin{array}{l}\text { Concentration } \\
(\text { mean } \pm \text { S.D. })(n M)\end{array}$ & Accuracy (\%) & $\begin{array}{l}\text { Precision } \\
\text { (R.S.D., \%) }\end{array}$ \\
\hline Irinotecan & & & & Irinotecan & & & \\
\hline 2.5 & $2.7 \pm 0.38$ & 108.0 & 14.1 & 5.0 & $4.3 \pm 0.56$ & 86.0 & 13.0 \\
\hline 250.0 & $268.5 \pm 24.2$ & 107.4 & 9.0 & 250.0 & $236.5 \pm 16.4$ & 94.6 & 6.9 \\
\hline 5000.0 & $5056 \pm 138.6$ & 101.1 & 2.7 & 5000.0 & $4890.0 \pm 264.0$ & 97.8 & 5.4 \\
\hline SN-38 & & & & SN-38 & & & \\
\hline 2.5 & $2.4 \pm 0.3$ & 96.0 & 12.5 & 5.0 & $4.5 \pm 0.65$ & 90.0 & 14.4 \\
\hline 250.0 & $235.0 \pm 18.7$ & 94.0 & 7.9 & 250.0 & $263.5 \pm 23.5$ & 105.4 & 8.9 \\
\hline 500.0 & $553 \pm 50.9$ & 110.6 & 9.2 & 500.0 & $515.0 \pm 31.8$ & 103.0 & 6.2 \\
\hline
\end{tabular}

S.D. = standard deviation; R.S.D. = relative standard deviation.

of irinotecan and SN-38 from the MCTS. A solution of $0.01 \mathrm{M}$ $\mathrm{HCl}$ :methanol $(2: 3, \mathrm{v} / \mathrm{v})$ was selected because it provided adequate specificity, sensitivity, and extracted the analytes in a lactone form, which is the cytotoxic form for both the prodrug and metabolite. The average extraction recoveries for irinotecan with this approach were $80.4 \%, 90.5 \%$, and $111 \%$ at low, medium, and high concentrations in MCTS extracts, and $89 \%$, $109.4 \%$, and $94.5 \%$ in culture medium. The mean recoveries for $\mathrm{SN}-38$ were $82.4 \%, 90.3 \%$, and $93.9 \%$ in MCTS extracts, and $111.6 \%, 95 \%$, and $105.2 \%$ in culture medium (Table 2).

\section{Stability}

A number of studies have been performed to determine the stability of irinotecan and SN-38 in methanol-based solutions and other matrices. The analytes have been shown to be stable after five freeze-thaw cycles. These compounds were also found to be stable up to $1 \mathrm{mo}$ at $4^{\circ} \mathrm{C}$ and at least 8 wk when stored at $-80^{\circ} \mathrm{C}[31,38]$. However, the lactone forms of irinotecan and $\mathrm{SN}-38$ were unstable during storage at room temperature or at $37^{\circ} \mathrm{C}$ for more than $20 \mathrm{~h}[38-40]$. Sample acidification overcomes this instability and allows determination of the analyte concentrations [31].

In this study, frozen QC samples either in MCTS extracts or medium stored at $-80^{\circ} \mathrm{C}$ were tested over a 31-d period. Irinotecan and SN-38 showed no sign of degradation. Acidified QC samples in both matrices were allowed to stand at room temperature for $12 \mathrm{~h}$ and maintained the nominal starting concentration.

\section{Analysis of Irinotecan and $S N-38$ in MCTS and Culture Medium}

The validated method was used to investigate the distribution of irinotecan and its active metabolite SN-38 in MCTS. MCTS were treated with $20.6 \mu \mathrm{M}$ irinotecan for various lengths of time and fractioned using the serial trypsinization protocol described above. The prodrug and metabolites were then extracted and analyzed with nLC-MS/MS.

\section{Quantification of Irinotecan in MCTS}

Data were processed and peak area ratios of irinotecan compared to camptothecin were calculated by using Quan Browser of Xcalibur software. Based on the slope and intercept of the irinotecan calibration curve, the concentrations of irinotecan extracted from cells deriving from the outer rim, intermediate, and core regions of MCTS were calculated. Exact amounts of the drug were then determined based on the total volume of each sample $(100 \mu \mathrm{L})$. The weight of the cells was used to normalize and quantify the final concentration of the drug $(\mu \mathrm{g} / \mathrm{g}$ cells $)$ in spatially distinct regions of MCTS.

Table 2. Percent Extraction Efficiency of Irinotecan and SN-38 from MCTS and Culture Medium Tested at Different Concentration Levels

\begin{tabular}{|c|c|c|c|c|c|c|c|}
\hline \multirow{2}{*}{$\begin{array}{l}\text { Nominal } \\
\text { concentration (nM) }\end{array}$} & \multicolumn{3}{|l|}{ MCTS extracts } & \multirow{2}{*}{$\begin{array}{l}\text { Nominal } \\
\text { concentration }(\mathrm{nM})\end{array}$} & \multicolumn{3}{|l|}{ Culture medium } \\
\hline & $\begin{array}{l}\text { Concentration } \\
(\text { mean } \pm \text { S.D. })(\mathrm{nM})\end{array}$ & Accuracy (\%) & $\begin{array}{l}\text { Precision } \\
\text { (R.S.D., \%) }\end{array}$ & & $\begin{array}{l}\text { Concentration } \\
(\text { mean } \pm \text { S.D. })(n M)\end{array}$ & Accuracy (\%) & $\begin{array}{l}\text { Precision } \\
\text { (R.S.D., \%) }\end{array}$ \\
\hline Irinotecan & & & & Irinotecan & & & \\
\hline 2.5 & $2.01 \pm 0.1$ & 80.4 & 4.9 & 5.0 & $4.45 \pm 0.52$ & 89.0 & 11.7 \\
\hline 250.0 & $226.3 \pm 32.6$ & 90.5 & 14.4 & 250.0 & $273.6 \pm 37.0$ & 109.4 & 13.5 \\
\hline 5000.0 & $5550 \pm 548.8$ & 111.0 & 9.9 & 5000.0 & $4725.5 \pm 396.2$ & 94.5 & 8.4 \\
\hline SN-38 & & & & SN-38 & & & \\
\hline 2.5 & $2.06 \pm 0.3$ & 82.4 & 14.6 & 5.0 & $5.58 \pm 0.78$ & 111.6 & 14.0 \\
\hline 250.0 & $225.8 \pm 28.5$ & 90.3 & 12.6 & 250.0 & $237.4 \pm 32.5$ & 95.0 & 13.7 \\
\hline 500.0 & $469.5 \pm 54.2$ & 93.9 & 11.5 & 500.0 & $525.8 \pm 45.9$ & 105.2 & 8.7 \\
\hline
\end{tabular}

S.D. $=$ standard; R.S.D. $=$ relative standard deviation 


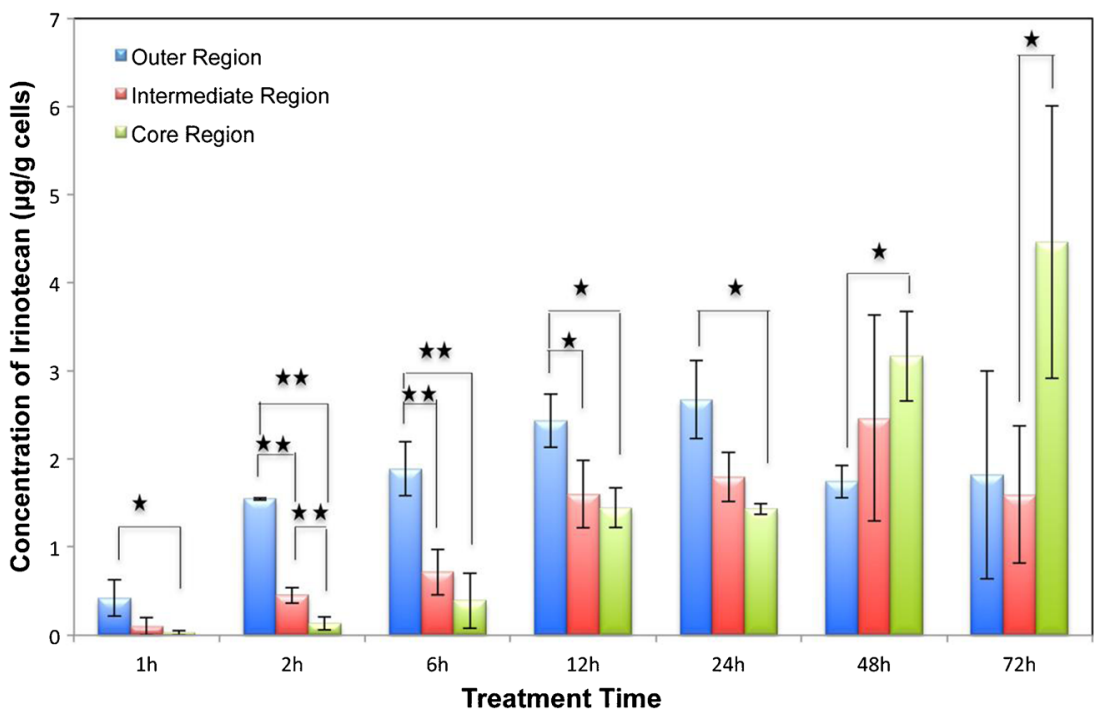

Figure 4. Irinotecan quantification in different regions of the MCTS. MCTS were treated with $20.6 \mu \mathrm{M}$ irinotecan for different length of time $(1,2,6,12,24,48,72 \mathrm{~h}) ; n=3$ replicates per time point, ${ }^{\star} P<0.05,{ }^{\star \star} P<0.01$

As shown in Supplemental Figure 4, the total amount of irinotecan accumulated in the MCTS increased with the drug incubation time. In the first $6 \mathrm{~h}$ of incubation, irinotecan accumulated primarily in the periphery region, followed by enhanced drug penetration into the central part of the MCTS within $24 \mathrm{~h}$ (Figure 4). After $24 \mathrm{~h}$ of treatment, irinotecan concentrations slightly decreased in the outer and intermediate regions while accumulating in the core area of the MCTS. We hypothesize that these changes could be due to irinotecan being cleared from cells via efflux transporters like P-glycoprotein (Pgp) [41-43], ABCG2/BCRP [7], and multidrug resistanceassociated protein (MRP) [44], which actively transport chemotherapy agents out of the cell, resulting in reduced concentration in MCTS. Metabolism is another factor that influences the parent drug amount in cells. For example, carboxylesterase activity for converting irinotecan into $\mathrm{SN}-38$ in proliferating and live cells is higher than in low $\mathrm{pH}$ and hypoxic cells, which would result in a prodrug accumulation in the core of MCTS
$[45,46]$. Moreover, under acidic conditions, the cytotoxic lactone form of irinotecan is favored, whereas at physiological or higher $\mathrm{pH}$, its inactive carboxylate form is favored [29-31]. The lactone forms are passively transported in cells, resulting in an uptake 10 times greater than the carboxylate forms [47]. Therefore, the gradient in MCTS with low $\mathrm{pH}$ in the necrotic core leads to the accumulation of irinotecan. Biotransformation of the drug into other metabolites like 7-ethyl-10- [4-N-(5-aminopentanoic acid)-1-piperidino]carbonyloxycamptothecin (APC) and 7-ethyl-10-(4-amino-1-piperidino) carbonyloxycamptothecin (NPC) also affects the compound amount being detected.

\section{Quantification of SN-38 in MCTS}

SN-38 was quantified with the same approach as irinotecan. Figure 5 illustrates the distribution of this active metabolite in MCTS. SN-38 concentration was quantifiable after $12 \mathrm{~h}$ of

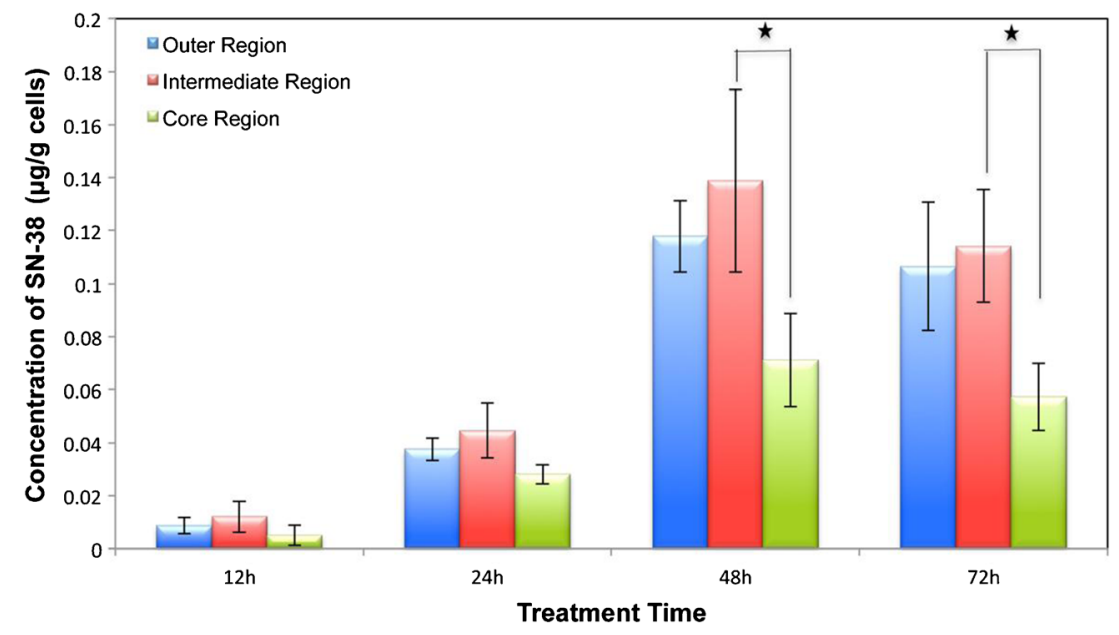

Figure 5. Quantification of the active metabolite SN-38 in different regions of the MCTS; $n=3$ replicates per time point, ${ }^{*} P<0.05$ 

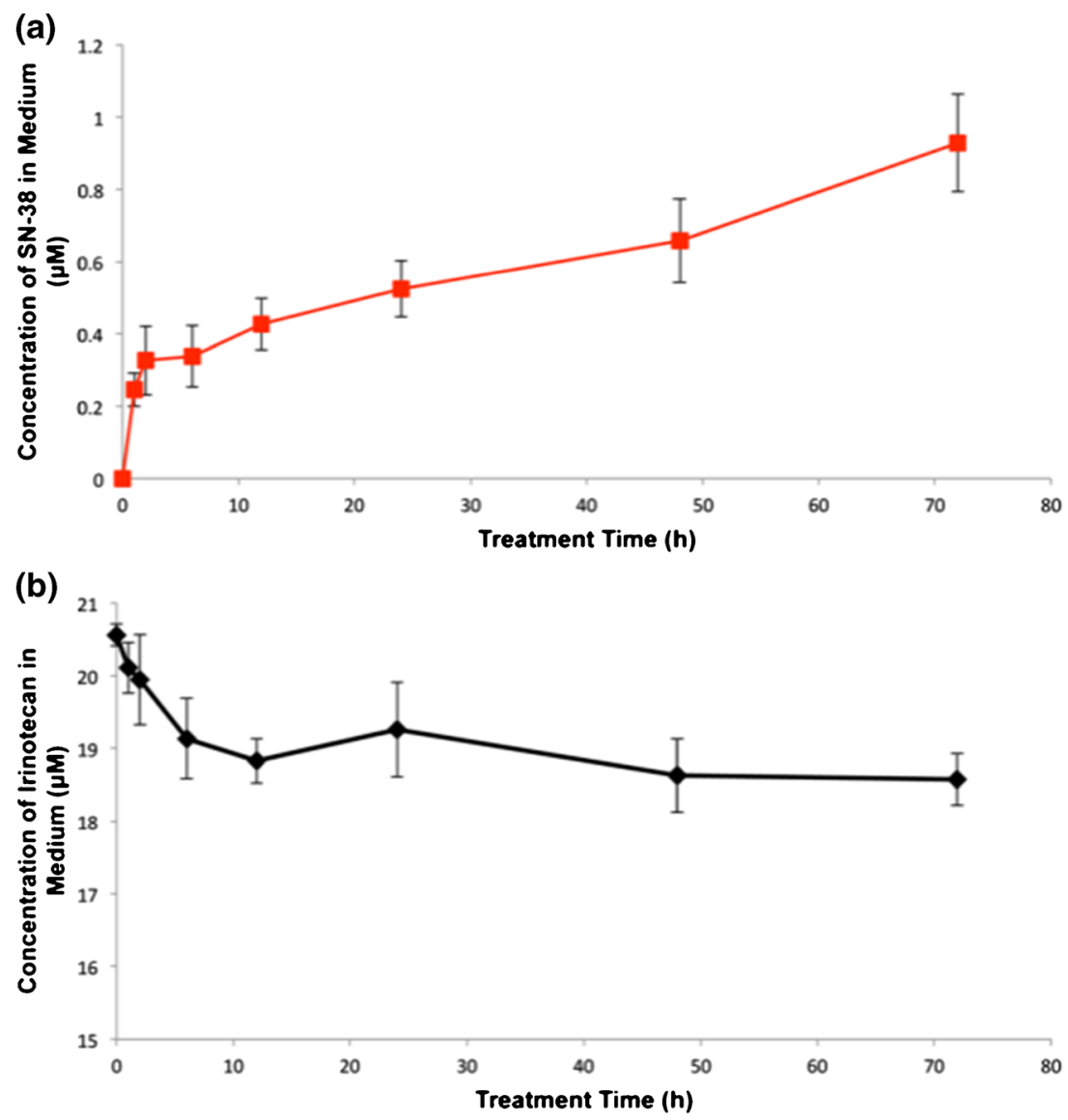

Figure 6. Quantification of irinotecan and SN-38 in culture medium collected at different time points after incubation of MCTS with irinotecan $(20.6 \mu \mathrm{M}) ; n=3$ replicates per time point

treatment and the concentration increases substantially at 48 and $72 \mathrm{~h}$. The total amount of SN-38 decreased about $15 \%$ after $48 \mathrm{~h}$ and it is about 30 times lower than the corresponding irinotecan level at $72 \mathrm{~h}$ (Supplemental Figure 4). Also, this metabolite is more abundant in outer and middle regions than in the core region. In spite of the lower conversion of SN-38 from the prodrug irinotecan in the necrotic core, efflux-based transport is another factor affecting its concentration and localization in MCTS.

In our previous studies using the MALDI IMS methodology, irinotecan was determined to have a time-dependent penetration pattern into MCTS. The prodrug showed an accumulation in the peripheral regions of MCTS after the first $6 \mathrm{~h}$, with increasing permeability into the center and accumulating in the

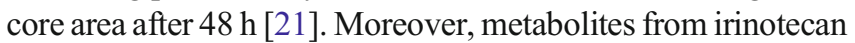
showed a higher abundance in the outer and intermediate regions of MCTS after irinotecan treatment. These MALDI imaging data are consistent with the LC-MS/MS results generated in the current study.

\section{Quantification of Irinotecan and SN-38 in Culture Medium}

Concentrations of irinotecan and SN-38 were quantified in culture medium at different treatment time points. Shown in
Figure $6 b$, concentration of irinotecan decreased about $9 \%$ during the 72-h treatment period because of accumulation of the drug in the MCTS. An increasing concentration of SN-38 in the culture medium was detected (Figure 6a), which indicates that irinotecan can be efficiently converted to its active metabolite $\mathrm{SN}-38$ by MCTS viable cells and exported into medium. The efflux of the metabolite can be a reason for the limited detection of the metabolite in MCTS before $12 \mathrm{~h}$ and slightly decreased intracellular SN-38 concentration at the $72 \mathrm{~h}$ time point.

In future studies, other key metabolites of irinotecan, like NPC and APC, could be investigated. APC and NPC are metabolites of irinotecan produced from catabolism of the prodrug by cytochrome $P 450$ [27]. NPC and APC are weak inhibitors of DNA Topo I and NPC can be converted to the active metabolite SN-38 [26, 27]. Analysis of these metabolites will also have great significance for a better understanding of drug delivery and metabolism in the heterogeneous cells in MCTS.

\section{Conclusions}

By blending 3D cell cultures, serial trypsinization, and $\mathrm{nLC}$ $\mathrm{MS} / \mathrm{MS}$, we are developing a novel methodology for accurate 
and simple evaluation of drug delivery and metabolism in vitro. This approach is quantitative and can be easily implemented for the spatial mapping of therapeutics, identifying and quantifying the novel metabolites, as well as providing preclinical information about their permeability properties. In this study, we demonstrated the utility of the method with the wellestablished drug irinotecan and its metabolite, SN-38; however, the promise of the approach lies in the testing of novel therapeutics.

\section{Acknowledgments}

The authors thank the Mass Spectrometry and Proteomics Facility at the University of Notre Dame and, in particular, the assistance of Dr. William Boggess. They also thank Dr. Susan Skube for assistance with manuscript editing. This research was supported by the National Science Foundation (CAREER Award CHE-1351595) for A.B...H. X.L. was supported by the Notre Dame Chemistry Biochemistry Biology Interface (CBBI) program and NIH Training Grant T32 GM075762. The Walther Cancer Foundation provided salary support for A.B...H.

\section{References}

1. Mullard, A.: 2013 FDA drug approvals. Nat. Rev. Drug Discov. 13, 85-89 (2014)

2. Hay, M., Thomas, D.W., Craighead, J.L., Economides, C., Rosenthal, J.: Clinical development success rates for investigational drugs. Nat. Biotechnol. 32, 40-51 (2014)

3. Tredan, O., Galmarini, C.M., Patel, K., Tannock, I.F.: Drug resistance and the solid tumor microenvironment. JNCI 99, 1441-1454 (2007)

4. Lammers, T., Kiessling, F., Hennink, W.E., Storm, G.: Drug targeting to tumors: principles, pitfalls and (pre-) clinical progress. J. Control. Release 161, 175-187 (2012)

5. Minchinton, A.I., Tannock, I.F.: Drug penetration in solid tumours. Nat. Rev. Cancer 6, 583-592 (2006)

6. Mehta, G., Hsiao, A.Y., Ingram, M., Luker, G.D., Takayama, S.: Opportunities and challenges for use of tumor spheroids as models to test drug delivery and efficacy. J. Controlled Release 164, 192-204 (2012)

7. Sarkadi, B., Homolya, L., Szakacs, G., Varadi, A.: Human multidrug resistance $\mathrm{ABCB}$ and $\mathrm{ABCG}$ transporters: participation in a chemoimmunity defense system. Physiol. Rev. 86, 1179-1236 (2006)

8. Pampaloni, F., Reynaud, E.G., Stelzer, E.H.K.: The third dimension bridges the gap between cell culture and live tissue. Nat. Rev. Mol. Cell Biol. 8, 839-845 (2007)

9. Vinci, M., Gowan, S., Boxall, F., Patterson, L., Zimmermann, M., Court, W., Lomas, C., Mendiola, M., Hardisson, D., Eccles, S.A.: Advances in establishment and analysis of three-dimensional tumor spheroid-based functional assays for target validation and drug evaluation. BMC Biol. 10, 29 (2011)

10. LaBarbera, D.V., Reid, B.G., Yoo, B.H.: The multicellular tumor spheroid model for high-throughput cancer drug discovery. Expert Opin. Drug Discov. 7, 819-830 (2012)

11. Mazzoleni, G., Di Lorenzo, D., Steimberg, N.: Modeling tissues in 3D: the next future of pharmaco-toxicology and food research? Genes Nutr. 4, 1322 (2009)

12. Hirschhaeuser, F., Menne, H., Dittfeld, C., West, J., Mueller-Klieser, W., Kunz-Schughart, L.A.: Multicellular tumor spheroids: an underestimated tool is catching up again. J. Biotechnol. 148, 3-15 (2010)

13. Sutherland, R.M., McCredie, J.A., Inch, W.R.: Growth of multicell spheroids as a model of nodular carcinomas. J. Nat. Cancer Inst. 46, 113-120 (1971)

14. Wartenberg, M., Hescheler, J., Acker, H., Diedershagen, H., Sauer, H.: Doxorubicin distribution in multicellular prostate cancer spheroids evaluated by confocal laser scanning microscopy and the 'Optical probe technique.'. Cytometry 31, 137-145 (1998)

15. Perche, F., Torchilin, V.P.: Cancer cell spheroids as a model to evaluate chemotherapy protocols. Cancer Biol. Ther. 13, 1205-1213 (2012)

16. Wang, X., Zhen, X., Wang, J., Zhang, J., Wu, W., Jiang, X.: Doxorubicin delivery to $3 \mathrm{D}$ multicellular spheroids and tumors based on boronic acidrich chitosan nanoparticles. Biomaterials 34, 4667-4679 (2013)

17. Bichay, T.J., Inch, W.R.: Resistance of V79 multicell spheroids to mitoxantrone: drug uptake and cytotoxicity. Cancer Drug Deliv. 4, 201-211 (1987)

18. West, G.W., Weichselbaum, R., Little, J.B.: Limited penetration of methotrexate into human osteosarcoma spheroids as a proposed model for solid tumor resistance to adjuvant chemotherapy. Cancer Res. 40, 3665-3668 (1980)

19. Wibe, E.: Resistance to vincristine of human cells grown as multicellular spheroids. Br. J. Cancer 42, 937-941 (1980)

20. Sutherland, R., Buchegger, F., Schreyer, M., Vacca, A., Mach, J.P.: Penetration and binding of radiolabeled anti-carcinoembryonic antigen monoclonal antibodies and their antigen binding fragments in human colon multicellular tumor spheroids. Cancer Res. 47, 1627-1633 (1987)

21. Liu, X., Weaver, E.M., Hummon, A.B...: Evaluation of therapeutics in three-dimensional cell culture systems by MALDI imaging mass spectrometry. Anal. Chem. 85(13), 6295-6302 (2013)

22. Freyer, J.P., Sutherland, R.M.: Selective dissociation and characterization of cells from different regions of multicell tumor spheroids. Cancer Res. $\mathbf{4 0}$, 3956-3965 (1980)

23. McMahon, K.M., Volpato, M., Chi, H.Y., Musiwaro, P., Poterlowicz, K., Peng, Y.: Characterization of changes in the proteome in different regions of 3D multicell tumor spheroids. J. Proteome Res. 11(5), 2863-2875 (2012)

24. Keithley, R.B., Weaver, E.M., Metzinger, M.P., Rosado, A.M., Hummon, A.B..., Dovichi, N.J.: Single cell metabolic profiling of tumor mimics. Anal. Chem. 85, 8910-8918 (2013)

25. Cunningham, D., Maroun, J., Vanhoefer, U., Van, E.: Optimizing the use of irinotecan in colorectal cancer. Oncologist 6(Suppl 4), 17-23 (2001)

26. $\mathrm{Xu}, \mathrm{Y} .$, Villalona-Calero, M.A.: Irinotecan: mechanisms of tumor resistance and novel strategies for modulating its activity. Ann. Oncol. 13, 1841-1851 (2002)

27. Vanhoefer, U., Harstick, A., Achterrath, W., Cao, A., Seeber, S., Rustum, Y.M.: Irinotecan in the treatment of colorectal cancer: clinical overview. J. Clin. Oncol. 19, 1501 (2001)

28. de Jong, F.A., Mathijssen, R.H., de Bruijn, P., Loos, W.J., Verweij, J., Sparreboom, A.: Determination of irinotecan (CPT-11) and SN-38 in human whole blood and red blood cells by liquid chromatography with fluorescence detection. J. Chromatogr., B 795, 383-388 (2003)

29. Khan, S., Ahmad, A., Guo, W.: A simple and sensitive LC/MS/MS assay for 7-ethyl-10-hydroxycamptothecin (SN-38) in mouse plasma and tissues: application to pharmacokinetic study of liposome entrapped SN-38 (LESN38). J. Pharm. Biomed. Anal. 37, 135-142 (2005)

30. Bardin, S., Guo, W., Johnson, J.L., Khan, S., Ahmad, A., Duggan, J.X., Ayoub, J., Ahmad, I.: Liquid chromatographic-tandem mass spectrometric assay for the simultaneous quantification of Camptosar and its metabolite $\mathrm{SN}-38$ in mouse plasma and tissues. J. Chromatogr. A 1073, 249-255 (2005)

31. D’Esposito, F., Tattam, B.N., Ramzan, I., Murray, M.: A liquid chromatography/electrospray ionization mass spectrometry (LC-MS/MS) assay for the determination of irinotecan (CPT-11) and its two major metabolites in human liver microsomal incubations and human plasma samples. J. Chromatogr., B 875, 522-530 (2008)

32. Hu, Z.P., Yang, X.X., Chen, X., Chan, E., Duan, W., Zhou, S.F.: Simultaneous determination of irinotecan (CPT-11) and SN-38 in tissue culture media and cancer cells by high performance liquid chromatography: application to cellular metabolism and accumulation studies. J. Chromatogr., B: Anal. Technol. Biomed. Life Sci. 850, 575-580 (2007)

33. Bansal, T., Awasthi, A., Jaggi, M., Khar, R.K., Talegaonkar, S.: Development and validation of reversed phase liquid chromatographic method utilizing ultraviolet detection for quantification of irinotecan (CPT-11) and its active metabolite, $\mathrm{SN}-38$, in rat plasma and bile samples: application to pharmacokinetic studies. Talanta 76, 1015-1021 (2008)

34. Zhang, W., Dutschman, G.E., Li, X., Ye, M., Cheng, Y.C.: Quantitation of irinotecan and its two major metabolites using a liquid chromatographyelectrospray ionization tandem mass spectrometric. J. Chromatogr., B: Anal. Technol. Biomed. Life Sci. 877, 3038-3044 (2009)

35. Chen, X., Peer, C.J., Alfaro, R., Tian, T., Spencer, S.D., Figg, W.D.: Quantification of irinotecan, SN38, and SN38G in human and porcine 
plasma by ultra high-performance liquid chromatography-tandem mass spectrometry and its application to hepatic chemoembolization. J. Pharm. Biomed. Anal. 62, 140-148 (2012)

36. Li, H., Hummon, A.B...: Imaging mass spectrometry of three-dimensional cell culture systems. Anal. Chem. 83, 8794-8801 (2011)

37. Godoy, P., Hewitt, N.J., Albrecht, U., Andersen, M.E., Ansari, N., Bhattacharya, S.: Recent advances in $2 \mathrm{D}$ and $3 \mathrm{D}$ in vitro systems using primary hepatocytes, alternative hepatocyte sources, and non-parenchymal liver cells and their use in investigating mechanisms of hepatotoxicity, cell signaling, and ADME. Arch. Toxicol. 87, 1315-1530 (2013)

38. de Bruijn, P., Verweij, J., Loos, W.J., Nooter, K., Stoter, G., Sparreboom, A.: Determination of irinotecan (CPT-11) and its active metabolite SN-38 in human plasma by reversed-phase high-performance liquid chromatography with fluorescence detection. J. Chromatogr., B 698, 277-285 (1997)

39. Sai, K., Kaniwa, N., Ozawa, S., Sawada, J.I.: An analytical method for irinotecan (CPT-11) and its metabolites using a high-performance liquid chromatography: parallel detection with fluorescence and mass spectrometry. Biomed. Chromatogr. 16, 209-218 (2002)

40. Sparreboom, A., de Bruijn, P., de Jonge, M.J., Loos, W.J., Stoter, G., Verweij, J.: Liquid chromatographic determination of irinotecan and three major metabolites in human plasma, urine and feces. J. Chromatogr. B Biomed. Sci. Appl. 712, 225-235 (1998)
41. Ramesh, M., Ahlawat, P., Srinivas, N.R.: Irinotecan its active metabolite, SN-38: review of bioanalytical methods and recent update from clinical pharmacology perspectives. Biomed. Chromatogr. 24, 104-123 (2010)

42. Bansal, T., Mishra, G., Jaggi, M., Khar, R.K., Talegaonkar, S.: Effect of Pglycoprotein inhibitor, verapamil, on oral bioavailability and pharmacokinetics of irinotecan in rats. Eur. J. Pharm. Sci. 36, 580-590 (2009)

43. Filipski, E., Berland, E., Ozturk, N., Guettier, C., van der Horst, G.T., Lévi, F., Okyar, A.: Optimization of irinotecan chronotherapy with Pglycoprotein inhibition. Toxicol. Appl. Pharmacol. 274(3), 471-479 (2014)

44. Norris, M.D., Smith, J., Tanabe, K., Tobin, P., Flemming, C., Scheffer, G.L., Wielinga, P., Cohn, S.L., London, W.B., Marshall, G.M., Allen, J.D., Haber, M.: Expression of multidrug transporter MRP4/ABCC4 is a marker of poor prognosis in neuroblastoma and confers resistance to irinotecan in vitro. Mol. Cancer Ther. 4, 547-553 (2005)

45. Matzow, T., Cowen, R.L., Williams, K.J., Telfer, B.A., Flint, P.J., Southgate, T.D., Saunders, M.P.: Hypoxia-targeted over-expression of carboxylesterase as a means of increasing tumour sensitivity to irinotecan (CPT11). J. Gene Med. 9(4), 244-252 (2007)

46. Strese, S., Fryknäs, M., Larsson, R., Gullbo, J.: Effects of hypoxia on human cancer cell line chemosensitivity. BMC Cancer 13, 331 (2013)

47. Kobayashi, K., Bouscarel, B., Matsuzaki, Y.: pH-dependent uptake of irinotecan and its active metabolite, SN-38, by intestinal cells. Int. J. Cancer 83, 491-496 (1999) 